\title{
ERBB2 juxtamembrane domain (trastuzumab binding site) gene mutation is a rare event in invasive breast cancers overexpressing the ERBB2 gene
}

\author{
Thaer Khoury ${ }^{1}$, Wilfrido Mojica ${ }^{2}$, David Hicks ${ }^{3}$, Petr Starostik ${ }^{1}$, Foluso Ademuyiwa ${ }^{4}$, \\ Bagirathan Janarthanan $^{5}$ and Richard T Cheney ${ }^{1}$ \\ ${ }^{1}$ Department of Pathology, Roswell Park Cancer Institute, Elm and Carlton Streets, Buffalo, NY, USA; \\ ${ }^{2}$ Department of Pathology, Kalieda Health System, Buffalo, NY, USA; ${ }^{3}$ Department of pathology, Rochester \\ general Hospital, Rochester, NY, USA; ${ }^{4}$ Department of Oncology, Roswell Park Cancer Institute, Buffalo, \\ NY, USA and ${ }^{5}$ Department of Oncology, Florida Hospital, Orlando, FL, USA
}

\begin{abstract}
The recent development of targeted therapies using monoclonal antibodies has added new dimensions to breast cancer treatment. Trastuzumab has been added to the regimens that contain chemotherapeutic agents, which has improved the clinical outcomes of patients in both the adjuvant and metastatic settings. However, trastuzumab resistance, both de novo and acquired, continues to be problematic. There have been scattered studies reporting ERBB2 gene mutation, but nothing is currently known about the ERBB2 binding site mutations. In the current study, we examined the ERBB2 juxtamembrane domain trastuzumab binding site for mutations in invasive breast cancers overexpressing ERBB2. Pure tumor cells of 54 breast cancer patients were procured using laser capture microdissection. Two polymerase chain reaction primer pairs were designed to amplify the trastuzumab binding site sequence. The polymerase chain reaction product was sequenced. Standard clinicopathological data were recorded. For the 54 patients, there was one (2\%) case that showed missense point mutation in exon 17 (H559A). There were nine patients treated with trastuzumab in the metastatic setting, none of which had gene mutation. Therefore, we conclude that ERBB2 juxtamembrane domain (trastuzumab binding site) gene mutation is a rare event in breast cancer. Although it is unclear whether this substitution would result in trastuzumab target therapy resistance, this would not account for the relatively high frequency of this resistance encountered clinically.

Modern Pathology (2011) 24, 1055-1059; doi:10.1038/modpathol.2011.64; published online 15 April 2011
\end{abstract}

Keywords: breast cancer; gene mutation; ERBB2; trastuzumab

Overexpression of the tyrosine kinase receptor ERBB2 (HER2) occurs in approximately $18-20 \%$ of early-stage invasive breast cancers. ${ }^{1,2}$ The ERBB2 receptor protein has an extracellular domain, a transmembrane domain, and an intracellular tyrosine kinase domain. ${ }^{3}$ Phosphorylation of key residues and the recruitment of cytoplasmic signaling molecules that subsequently initiate signaling

Correspondence: Dr T Khoury, MD, Department of Pathology, Roswell Park Cancer Institute, Elm and Carlton streets, Buffalo, NY 14263, USA.

E-mail: thaer.khoury@roswellpark.org

Received 11 October 2010; revised 17 February 2011; accepted 18

February 2011; published online 15 April 2011 cascades occur through dimerization of the ERB receptors.

Increasingly, management decisions regarding whether oncologists should prescribe adjuvant treatment are being based on the biology of the patient's tumor, with a consideration of the hormone receptor status, ${ }^{4}$ and more recently, with the assessment of ERBB2. ${ }^{5}$ The identification of the amplification of ERBB2 gene and the overexpression of its protein product has resulted in targeted monoclonal antibody-based therapy. ${ }^{6,7}$ Preclinical studies indicate synergistic anti-tumor activity when trastuzumab is combined with a number of anti-cancer drugs. Additive cytotoxic interactions between trastuzumab and other agents, including paclitaxel, 
also have been shown. ${ }^{8}$ However, several patients who initially received trastuzumab in the adjuvant setting develop recurrent tumor and resistance within 1 year in the metastatic setting. ${ }^{9}$ This resistance has become a major clinical concern. There is no clinically verified factor that can be used to predict trastuzumab resistance. Several molecular mechanisms contributing to trastuzumab resistance have been proposed. ${ }^{10}$

The crystal structure of the ERBB2 receptor by itself and in combination with trastuzumab was deduced. The receptor has an extracellular region composed of 630 amino acids, a single membranespanning region and a cytoplasmic tyrosine kinase. The extracellular region is composed of four domains. These four domains are arranged as tandem repeats of a two-domain unit consisting of an L domain (domains I and III; 190 amino acids each) and a cysteine-rich domain (domains II and IV; $\sim 120$ amino acids each). Trastuzumab binds to ERBB2 receptor to a region in domain IV that is occupied by the domain II finger-like projection in other HER receptors. By crystallography, the antigen-antibody interaction is mediated by three regions along the C-terminal portion of domain IV. The first region is a loop formed by amino acid residues 557-561, the second from residues 570573, and the third from residues 593-603. The coding region of the first two residues is present in Exon 17 and the third residue is present in Exon 18. The antigen-antibody binding interactions formed by the first and third loop are mainly electrostatic, whereas the second is predominantly hydrophobic. ${ }^{11}$ The ability of trastuzumab to bind to this region correlates to its efficacy. It is postulated that bound antibody may achieve its therapeutic effect through several mechanisms, which may include, but are not limited to, antibody-dependent cellmeditated cytotoxicity, G1 arrest, receptor downregulation, or the prevention of heterodimer formation. ${ }^{12-14}$ However, to be efficacious, the therapeutic antibody must be able to bind to the receptor. The binding of an antibody to an antigen is entirely dependent upon non-covalent interactions. Small changes in the antigen structure can profoundly affect the strength of the antibody-antigen interaction. The loss of a single hydrogen bond at the interface can reduce the strength of interaction over a 1000-fold. Hence, changes in the ERBB2 sequence can potentially affect the binding efficacy of trastuzumab.

The structure of the ERBB2 receptor and its relationship to trastuzumab theoretically may have a role in de novo or acquired resistance. No study to date has tested the antibody interacting binding site of the juxtamembrane domain for possible mutations and correlated them with clinical evidence of resistance or recurrence in humans. The intent of this study is to analyze the critical regions of the $E R B B 2$ juxtamembrane domain trastuzumab binding site for mutations.

\section{Materials and methods}

\section{Cases Selection}

Patients diagnosed with invasive breast cancer that overexpressed ERBB2, detected by fluoresce in situ hybridization, were identified in Roswell Park Cancer Institute during the time frame of 19972004. Tissue samples from these patients were present in the form of archival, formalin-fixed paraffin-embedded material. There were 556 invasive breast cancer cases. ERBB2 overexpression was demonstrated by fluoresce in situ hybridization in $132(24 \%)$ cases. Microscopic evaluation of tissue sections demonstrated enough tumor cells for laser capture microdissection in 106 cases. Polymerase chain reaction rendered enough extracted DNA identified through spectrophotometry using NanoDrop in $54(53 \%)$ cases.

\section{Laser Capture Microdissection, Polymerase Chain Reaction, and Sequencing}

An appropriate slide and block from the identified cases are selected. Five-micron thick sections are cut and placed onto a slide, deparaffinized, and lightly stained with hematoxylin and eosin, followed by dehydration. Tumor cells were selectively procured from the slide by an Arcturus PixCell II LCM. At least 2000 pulses with size of $10 \mu$ were applied. The tumor cells were then lysed and the DNA isolated by a commercially available spin column kit, designed specifically for small quantities (PinPoint Isolation kit, Zymo Research). Polymerase chain reaction was performed using primers that encompass the nucleotides that correspond to amino-acid residues $557-561,570-573$, and 593-603. As the DNA is being isolated from formalin-fixed tissue, which is notorious for degrading DNA into small fragments of $<300 \mathrm{bp}$, and as the distance between amino-acid residue 557 and 603 correspond to over $900 \mathrm{bp}$, two primer pairs were designed. One primer pair encompassed the nucleotides corresponding to amino-acid residues 557-561 and 570-573 (forward primer 5'-CCGAGTACTGCAGGGGTATG-3'; reverse primer $5^{\prime}$-CTCACCGGTCCAAAACAGGT- ${ }^{\prime}$ ) and the other primer pair encompassed the nucleotides corresponding to amino acid residues 593-603 (forward primer 5'-ACAAAGGGGACCCAACTAA$3^{\prime}$; reverse primer 5'-GGCATGTAGGAGAGGTCA GG-3'). The former primer pair produced a 192-bp amplicon, whereas the latter primer pair produced a 186-bp amplicon. Polymerase chain reaction for both primer pairs utilized a modified hot start at $95^{\circ} \mathrm{C}$ for 2 min followed by 35 cycles with denaturation at $95^{\circ} \mathrm{C}$ for $15 \mathrm{~s}$, annealing at $54^{\circ} \mathrm{C}$ for $15 \mathrm{~s}$, and extension at $72^{\circ} \mathrm{C}$ for $30 \mathrm{~s}$. A final extension phase at $72^{\circ} \mathrm{C}$ for $10 \mathrm{~min}$ ended the reaction. A small amount of the PCR product was then visualized on an ethidium bromide-stained $2 \%$ gel in $100 \mathrm{~V}$ field. All amplicons were sequenced using Big Dye Terminator Sequencing (ABI PRISM 7700). 


\section{Results}

\section{Patients Characteristics}

Patients' age ranged from 35 to 90 years (median 58). There were $45(83 \%)$ ductal, $4(7.5 \%)$ lobular, 4 $(7.5 \%)$ mixed ductal and lobular, and $1(2 \%)$ micropapillary subtypes. The tumor was located in the right breast in $23(43 \%)$ cases, left breast in 30 $(55 \%)$ cases, and bilaterally in one $(2 \%)$ case. Modified Scarff Bloom Richardson grading was grade I in 5 (9\%), grade II in $16(30 \%)$, and grade III in $33(61 \%)$ cases. Lymph nodes (LN) metastasis was absent in $25(46 \%)$ cases (clinically in 10, and pathologically in 15 cases). Lymph nodes metastasis was identified in 1 to $3 \mathrm{LNs}$ in $11(20 \%)$ cases, 4 to 9 LNs in $10(19 \%)$ cases, and >9 LNs in $8(15 \%)$ cases. Tumor size was recorded in all cases but one. It was $\leq 1 \mathrm{~cm}$ in $33(62 \%)$ cases, $1.1-2 \mathrm{~cm}$ in 16 $(30 \%)$ cases, and $>2 \mathrm{~cm}$ in $4(8 \%)$ cases. Ductal carcinoma in situ was absent in $11(20 \%)$ cases, present in $\leq 50 \%$ of tumor size in $36(67 \%)$ cases, and $>50 \%$ of tumor size in $7(13 \%)$ cases. Hormonal receptors including estrogen receptor and progesterone receptor were recorded. Although hormonal receptor was absent in $22(41 \%)$ for ER and 31 (57\%) for PR, both hormones were absent simultaneously in $21(39 \%)$ cases. Local and/or distal metastasis was developed in 13 of $42(31 \%)$ cases. After tumor recurrence, trastuzumab therapy was provided for 10 patients. Therapy was discontinued in one patient because of therapy-related side effects (Table 1).

\section{ERBB2 Binding-Site Sequencing Results}

One of $54(2 \%)$ cases showed missense point mutation coding amino-acid residue 559, substituting histidine by arginine amino acid at residue 559 (H559A).

\section{Clinical History of the Patient with Mutation}

The patient is 76-year-old postmenopausal female with high-grade triple-positive (estrogen receptor/ progesterone receptor/ERBB2) ducal carcinoma, measuring $1.1 \mathrm{~cm}$ and with negative lymph nodes. Postoperatively, she received a course of radiation therapy, followed by a 5-year course of oral tamoxifen. No trastuzumab was given. The patient was disease-free after 5-year follow-up.

\section{Discussion}

There is no clinically verified factor that can be used to predict trastuzumab resistance in breast cancer. There are many proposed mechanisms of resistance including reduction of antibody affinity, and binding due to MUC4 overexpression was related to trastuzumab resistance in cell lines. ${ }^{15,16}$ Downstream signaling pathway members were proposed
Table 1 Clinicopathological characteristics of 54 cases

\begin{tabular}{|c|c|}
\hline Variable & Results \\
\hline Age, range (median) (in years) & $35-90(58)$ \\
\hline Tumor type (total) & 54 \\
\hline Ductal, $n(\%)$ & $45(83)$ \\
\hline Lobular, n (\%) & $4(7.5)$ \\
\hline Mixed lobular and ductal, n (\%) & $4(7.5)$ \\
\hline Micropapillary, n (\%) & $1(2)$ \\
\hline Laterality (total) & 54 \\
\hline Right, $n(\%)$ & $23(43)$ \\
\hline Left, $n(\%)$ & $30(56)$ \\
\hline Bilateral, $n(\%)$ & $1(2)$ \\
\hline SBR grade (total) & 54 \\
\hline $\mathrm{I}, n(\%)$ & $5(9)$ \\
\hline $\mathrm{II}, n(\%)$ & $16(30)$ \\
\hline III, $n(\%)$ & $33(61)$ \\
\hline Lymph node metastasis (total) & 54 \\
\hline No, $n(\%)$ & $25(46)^{a}$ \\
\hline $\mathrm{N} 1, n(\%)$ & $11(20)$ \\
\hline $\mathrm{N} 2, n(\%)$ & $10(19)$ \\
\hline N3, $n(\%)$ & $8(15)$ \\
\hline Size (total) & $53^{\mathrm{b}}$ \\
\hline T1a, $n(\%)$ & $3(6)$ \\
\hline T1b, $n(\%)$ & $7(13)$ \\
\hline T1c, $n(\%)$ & $23(43)$ \\
\hline Total T1, $n(\%)$ & $33(62)$ \\
\hline $\mathrm{T} 2, n(\%)$ & $16(30)$ \\
\hline $\mathrm{T} 3, n(\%)$ & $4(8)$ \\
\hline DCIS (total) & 54 \\
\hline $0 \%, n(\%)$ & $11(20)$ \\
\hline $1-50 \%, n(\%)$ & $36(67)$ \\
\hline$>50 \%, n(\%)$ & $7(13)$ \\
\hline ER (total) & 54 \\
\hline $0 \%, n(\%)$ & $22(41)$ \\
\hline $1-50 \%, n(\%)$ & $8(15)$ \\
\hline $51-100 \%, n(\%)$ & $24(44)$ \\
\hline PR (total) & 54 \\
\hline $0 \%, n(\%)$ & $31(57)$ \\
\hline $1-50 \%, n(\%)$ & $13(24)$ \\
\hline $51-100 \%, n(\%)$ & $10(19)$ \\
\hline ER/PR (total) & 54 \\
\hline Both $0 \%, n(\%)$ & $21(39)$ \\
\hline At least one positive, $n(\%)$ & $33(61)$ \\
\hline Distant metastasis & $50^{\mathrm{c}}$ \\
\hline Mo, $n(\%)$ & $38(76)$ \\
\hline M1, n (\%) & $12(24)$ \\
\hline Transtuzumab therapy & $10^{\mathrm{d}}$ \\
\hline With response, $n(\%)$ & $6(67)$ \\
\hline With no response, $n(\%)$ & $3(33)$ \\
\hline
\end{tabular}

${ }^{\mathrm{a}} \mathrm{LN}$ metastasis was absent clinically in 10 cases and pathologically in 15 cases.

${ }^{\mathrm{b}}$ Size not documented in one case.

${ }^{\mathrm{c}}$ In four cases, evaluation for local or distant metastasis was not available.

${ }^{\mathrm{d}}$ One patient did not tolerate therapy.

as potential source for trastuzumab resistance, including p27 Kip1, PTEN, PI3K, mTOR, and Akt. ${ }^{17-19}$ Cross-talk with other signaling pathways including insulin-like growth factor receptor-1, ER pathway, and vascular endothelial growth factor that have the ability to bypass ERBB2 blockade have also been proposed to be involved in trastuzumab resistance. ${ }^{20-22}$ However, these studies were all preclinical or in cell lines, and to date, there are no clinically validated markers to predict de novo or 
acquired resistance to ERBB2-targeted therapy. We have recently proposed a gene signature that could predict trastuzumab resistance and worse clinical outcome. ${ }^{23}$ However, another possibility could be the binding site of the juxtamembrane domain.

Previous studies have searched for ERBB2 mutations in breast tumors and cell lines using a candidate mutation approach, sequencing of pooled amplicons, or sequencing of individual ERBB2 alleles from tumors with gene amplification. Stephens et $\mathrm{al}^{24}$ sequenced the complete ERBB2 coding sequence in 173 tumors, 18 of which were breast, and the kinase domain in 303 cancers (56 breast) and 235 cell lines ( 9 breast, none of those sequenced in this report). No mutations were found in any of the breast tumors or cell lines, and amplification status in the breast tumors and cell lines was not indicated. Lee et $a l^{25}$ used single-stranded conformation polymorphism to look for mutations in exons 18-23, which encode for the ERBB2 kinase domain, in 378 tumors of breast, colorectal, and gastric. Mutations were found in $4.3,2.9$, and $5 \%$ of cases, respectively. However, none of breast cancer cases had ERBB2 amplification. Gori $\mathrm{S}$ et al ${ }^{26}$ have sequenced exons 19-22, which encode the kinase domain of 41 patients with metastatic breast carcinoma and treated with trastuzumab. Two insertions in exon 20 (P780-H781insC) and one missense mutation in exon 21 (S856P) were found. Zito CI et $a I^{27}$ have sequenced the whole ERBB2 gene in four breast cancer cell lines and in one ovarian cell line. They found deletion in the coding region of exon 5 and 4 non-syn substitutions, one in exon 7 (C295X), two in exon 17 (I655V), and one in exon 22 (A890S).

None of these studies had specifically targeted the binding site of trastuzumab. Given the discovery of the crystal structure of ERBB2 receptor, illustrating the binding site with trastuzumab ${ }^{11}$ and the mystery of trastuzumab resistance, we thought of specifically targeting the trastuzumab binding site, looking for possible mutation that could explain this resistance.

In our previous report, we documented presence of mutation in 20 of 54 cases using temperature gradient capillary electrophoresis. ${ }^{28}$ However, sequencing these cases along with temperature gradient capillary electrophoresis-negative cases showed only one missense mutation. This mutation had resulted in substituting histidine by arginine amino acid at residue 559 (H559A). Arginine and histidine share some properties, including being both hydrophobic and charged. However, they differ in the side chain, van der Waals volume (148 vs 118), and the later being aromatic. It is unclear whether these differences make any significant change in the binding properties of the ERBB2 receptor and trastuzumab, or modify the function of the ERBB2 receptor. It would be important to define the exact nature of mutation, and later investigate the ERBB2 protein and trastuzumab interaction, through possibly protein crystallography. However, the only patient who carries this mutation did well, with at least 5-year disease-free without trastuzumab therapy.

In the studied cases, only patients who developed tumor recurrence were treated with trastuzumab. Overall, 13 patients had tumor recurrence, 9 of which had been treated with trastuzumab. None of these patients had binding site gene mutation. Therefore, we conclude that even if a binding site gene mutation exists and is consistently repetitive and not merely due to chance, and truly results in trastuzumab resistance, it cannot be accounted for the high frequency of trastuzumab resistance.

We conclude that a greater understanding of the biological heterogeneity within the ERBB2 positive cohort of breast cancer patients, particularly related to trastuzumab resistance is needed. Our data suggests that gene mutations in the ERBB2 juxtamembrane domain (trastuzumab binding site) is a rare event in breast cancer and is unlikely to account for the relatively high frequency of therapeutic resistance encountered clinically. Further studies that explore the relationship between tumor biology and response to adjuvant cytotoxic chemotherapy and ERBB2-targeted therapy are needed to help with the selection of optimal treatment regimens. We think that the proper direction for exploring the biology of trastuzumab resistance would be looking for a specific predictive gene signature. ${ }^{23,29}$

\section{Acknowledgement}

We thank the Alpha Foundation for the financial support.

\section{Disclosure/conflict of interest}

The authors declare no conflict of interest.

\section{References}

1 Yaziji H, Goldstein LC, Barry TS, et al. HER-2 testing in breast cancer using parallel tissue-based methods. JAMA 2004;291:1972-1977.

2 Owens MA, Horten BC, Da Silva MM. ERBB2 amplification ratios by fluorescence in situ hybridization and correlation with immunohistochemistry in a cohort of 6556 breast cancer tissues. Clin Breast Cancer 2004;5:63-69.

3 Olayioye MA, Neve RM, Lane HA, et al. The ErbB signaling network: receptor heterodimerization in development and cancer. EMBO J 2000;19:3159-3167.

4 Harvey JM, Clark GM, Osborne CK. Estrogen receptor status by immunohistochemistry is superior to the ligand-binding assay for prediction response to adjuvant endocrine therapy in breast cancer. J Clin Oncol 1999;17:1474-1481.

5 Cobleigh MA, Vogel CL, Tripathy D. Multinational study of the efficacy and safety of humanized antiERBB2 monoclonal antibody in women who have ERBB2-overexpressing metastatic breast cancer that 
has progressed after chemotherapy for metastatic disease. J Clin Oncol 1999;17:2639-2648.

6 Hancock MC, Langton BC, Chan T. A monoclonal antibody against the C-erb-2 protein enhances the cytotoxicity of cis-diamminedichloroplatinum against human breast and ovarian tumor cell lines. Cancer Res 1991;51:4575-4580.

7 Stancovski I, Hurwitz E, Leitner O. Mechanistic aspects of the opposing effects of monoclonal antibodies to the ERBB2 receptor on tumor growth. Proc Natl Acad Sci USA 1991;88:8691-8695.

8 Pegram MD, Konecny GE, O’Callaghan C, et al. Rational combinations of trastuzumab with chemotherapeutic drugs used in the treatment of breast cancer. J Natl Cancer Inst 2004;96:739-749.

9 Nahta R, Esteva FJ. Herceptin. Mechanisms of action and resistance. Cancer Lett 2006;232:123-138.

10 Normanno N, Maiello MR, De Luca A. Epidermal growth factor receptortyrosine kinase inhibitors (EGFR-TKIs): simple drugs with a complex mechanism of action? J Cell Physiol 2003;194:13-19.

11 Cho HS, Mason KR, Amyar KX, et al. Structure of the extracellular region of ERBB2 alone and in complex with the Herceptin. Nature 2003;421:756-760.

12 Cooley S, Burns LJ, Repka T, et al. Natural killer cell cytotoxicity of breast cancer targets is enhanced by two distinct mechanisms of antibody-dependent cellular cytotoxicity against LFA-3 and ERBB2/neu. Exp Hematol 1999;27:1533-1541.

13 Sliwokowski MX, Lofgren JA, Lewis GD. Nonclinical studies addressing the mechanism of action of trastuzumab (Herceptin). Semin Oncol 1999;26:60-70.

14 Baselga J, Albanell J. Mechanism of action of anti-ERBB2 monoclonal antibodies. Ann Oncol 2001;12:35-41.

15 Moniaux N, Andrianifahanana M, Brand RE, et al. Multiple roles of mucins in pancreatic cancer, a lethal and challenging malignancy. Br J Cancer 2004;91: 1633-1638.

16 Carraway KL, Perez A, Idris N, et al. Muc4/sialomucin complex in the mammary gland and breast cancer. J Mammary Gland Biol Neoplasia 2001;6:323-337.

17 Nahta R, Takahashi T, Ueno NT, et al. P27Kip1 downregulation is associated with trastuzumab resistance in breast cancer cells. Cancer Res 2004;64:3981-3986.

18 Nagata Y, Lan KH, Zhou X, et al. PTEN activation contributes to tumor inhibition by trastuzumab, and loss of PTEN predicts trastuzumab resistance in patients. Cancer Cell 2004;6:117-127.
19 Lu CH, Wyszomierski SL, Tseng LM, et al. Preclinical testing of clinically applicable strategies for overcoming trastuzumab resistance caused by PTEN deficiency. Clin Cancer Res 2007;13:5883-3888.

20 Nahta R, Yuan LXH, Zhang B, et al. Insulin-like growth factor-I receptor/ human epidermal growth factor receptor 2 heterodimerization contributes to trastuzumab resistance of breast cancer cells. Cancer Res 2005;65:11118-11128.

21 Chung Y-L, Sheu M-L, Yang S-C, et al. Resistance to tamoxifen-induced apoptosis is associated with direct interaction between ERBB2/neu and cell membrane estrogen receptor in breast cancer. Int J Cancer 2002;97:306-312.

22 du Manoir JM, Francia G, Man S, et al. Strategies for delaying or treating in vivo acquired resistance to trastuzumab in human breast cancer xenografts. Clin Cancer Res 2006;12:904-916.

23 Khoury T, Kanehira K, Wang D, et al. Breast carcinoma with amplified ERBB2: a gene expression signature specific for trastuzumab resistance and poor prognosis. Mod Pathol 2010;23:1364-1378.

24 Stephens P, Hunter C, Bignell G, et al. Lung cancer: intragenic ERBB2 kinase mutations in tumours. Nature 2004;30:525-526.

25 Lee JW, Soung YH, Seo SH, et al. Somatic mutations of ERBB2 kinase domain in gastric, colorectal, and breast carcinomas. Clin Cancer Res 2006;12:57-61.

26 Zito CI, Riches D, Kolmakova J, et al. Direct resequencing of the complete ERBB2 coding sequence reveals an absence of activating mutations in ERBB2 amplified breast cancer. Genes Chromosomes Cancer 2008;47: 633-638.

27 Gori S, Ludovini V, Colozza M, et al. ERBB2, EGFR, PIK3CA mutations in ERBB2+ metastatic breast cancer (MBC) patients (pts) treated with trastuzumab (T): incidence and correlation with response. Clin Oncol 2008;26(suppl): Abstract 1031.

28 Khoury T, Mojica W, Hicks DG, et al. Trastuzumab binding site mutations in ERBB2 positive invasive breast cancer: a potential predictor of less aggressive disease and trastuzumab resistance. Breast Cancer Res Treat 2006;100(Suppl 1):S31 (Abstract 308).

29 Staaf J, Ringnér M, Vallon-Christersson J, et al. Identification of subtypes in human epidermal growth factor receptor 2-positive breast cancer reveals a gene signature prognostic of outcome. J Clin Oncol 2010;10: 1813-1820. 